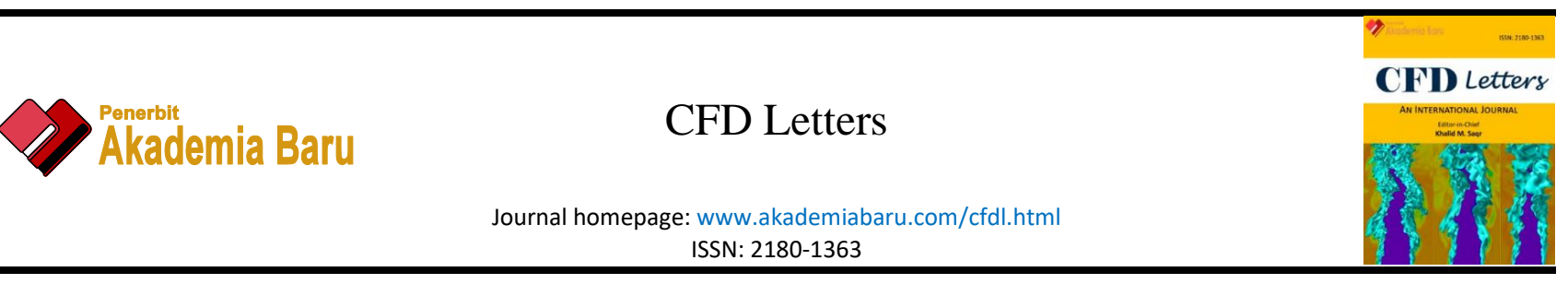

\title{
A Fundamental CFD Investigation of Offshore Structures for Artificial Coral Reef Development
}

\author{
Mohd Asamudin A. Rahman ${ }^{1}$, Muhammad Nadzrin Nazri ${ }^{1}$, Ahmad Fitriadhy ${ }^{1}$, Mohammad Fadhli \\ Ahmad $^{1}$, Erwan Hafizi Kasiman², Mohd Azlan Musa ${ }^{1}$, Fatin Alias ${ }^{1}$, Mohd Hairil Mohd ${ }^{1}{ }^{*}$ \\ Faculty of Ocean Engineering Technology and Informatics, Universiti Malaysia Terengganu, 21030 Kuala Nerus, Terengganu, Malaysia \\ School of Civil Engineering, Faculty of Engineering, Universiti Teknologi Malaysia, 81310 Skudai, Johor, Malaysia
}

\section{ARTICLE INFO}

\section{Article history:}

Received 21 May 2020

Received in revised form 20 July 2020

Accepted 25 July 2020

Available online 31 July 2020
Keywords:

Rigs-to-reefs; artificial reef; CFD simulation; upwelling; back eddy

\section{ABSTRACT}

Rigs-to-Reefs (R2R) is an alternative for oil and gas industry for decommissioning purpose. The program will benefit marine life as an artificial reef for a sustainable solution to promote ecosystem growth. For any oil platform considered for reefing, an extensive study on the suitability and performance of the artificial reef is essential. These studies will evaluate the stability and relevance of the structure as an artificial reef when deployed on the seabed. This paper presents the research on dynamic response and flow characteristics of a conceptual proposed R2R platform. The Computational Fluid Dynamics (CFD) simulation is intended to study the dynamic responses and flow characteristics analysis of a jacket oil platform for optimal settlement and growth of corals. Artificial reef performances were evaluated by calculating the performance indices of the upwelling and back eddy profile. It was found that the upwelling efficiency index is higher at current direction normal to the platform due to the higher frontal area exposed to the incoming flow. Meanwhile, back eddy efficiency index shows a significant low value for all directions. The CFD results presented will be discussed in terms of the possibility of better performance of an artificial reef which considering engineering and biological aspects

\section{Introduction}

In drastic and rampant global climate change, rise in marine environment pollution, and overexploited marine activities has impacted the marine ecosystem. In response to the urgent situation, many countries have taken the initiative in marine habitat restoration [1]. Since 2008, more than 50 countries participated in artificial coral reef construction [2]. Conventionally, offshore oil rig structures are decommissioned at the end of the platform production life. Decommissioning is a process of terminating the operations of the platform by means of dismantling and removal. There are several options for decommissioning depending on the legislation which is deep water disposal,

\footnotetext{
* Corresponding author.

E-mail address: m.hairil@umt.edu.my (Mohd Hairil Mohd)
} 
partial removal, complete removal, abandoning in place and for development of an artificial reef (AR). The environmental impact varies with the selection of the type of decommissioning. Studies show that "abandoning in place" has the least environmental impact in comparison to its counterparts [3]. Rig-to-Reef (R2R) which by means of reutilizing oil rig platforms as spatial ground provides not only biodiversity and habitat for biological communities but also a reduction in decommissioning cost [3-5].

The essence of converting oil rigs into artificial reefs is to increase habitats in the offshore environment; which subsequently lead to the increment in biomass for ocean productivity. In the case of rigs to reefs (R2R), settlement of scleractinian corals is among the critical indicators for the establishment of new habitats [6]. In general, larvae substratum selection is one of the crucial factors that regulate larvae settlement in the sea. In the offshore environment, when a new colony is established on newly introduced artificial reefs, substrate-borne vibration becomes one of the crucial parameters that determine successful community colonization [7]. In order for the structure (artificial reefs) to harbour a broad spectrum of marine organisms, the structure itself must provide a certain degree of stability for the biological community to thrive [8]. Numerous types of marine organism can be found on their submerged member after a certain period on any structure built offshore. Their distributions on the structural members differ by several factors including geographical location, water depth, water temperature and season, ocean current, platform design and operation.

Marine propagules can be into two types based on its motility, motile and non-motile. Tay et al., [9] stated that the travelling or the propagation speed of coral larva is not significant in comparison to the tidal current since the net distance of coral larvae is low Studies carried out by Abelson \& Denny [10] shows that marine larvae could be dissipated by laminar and turbulent flow regime. Four stages of the delivery method were defined for laminar which are direct interception, inertial impaction, gravitational deposition and the diffusional deposition. The characterization of turbulent flow in space and time is basically alternating of "ejections" and "sweeps" near the seabed [11].

Pressure distribution around the AR must be low to secure the attachment of coral larvae and increase the settlement success rate due to the larvae exploration at the surface of the AR [12]. Logically high-pressure distribution at the AR surface can 'sweep away' all the exploring larvae. When the larvae encounter any AR surface, the next step is either direct attach or exploration. The ability of the propagule to control its movement is crucial in order to ensure they have ample time to search for a suitable site to settle. The adhesive strength of these bio-fouling organism indicates the ability of the organism to withstand the pressure on the structure up to some extent [13].

Any AR deployed will be exposed to the hydrodynamic forces (drag and lift) in which affect the propagule on the surface. The velocity gradient on the surface tends to remove the propagule from the surface due to torque or rotary force exerted [14]. Reef stability and performance normally affected by current flow. One way to measure the performance of the reef is through the wake region developed behind the AR. Recirculation flow develops behind the bluff body is defined as the wake region caused by surrounding current profile. It has been suggested by numerous previous studies on AR that the wake region provides the marine life home or shelter as well as a feeding area. Figure 1 shows the wake region area, which can be divided into two regions, upwelling and back eddy. The formation of the upwelling and back eddy is subjected to the changes of the wake due to its shape, current profile, and seabed conditions. The turbulent flow over the AR promotes the upwelling region, which provides more nutrient into the water column. The enhanced nutrient subsequently promotes the production of marine life. It has been reported in the study by Wang et al., [15] on the influence of the AR shapes on the upwelling speed and profile.

Wake region performance and AR effectiveness can be measured using different approaches. It was reported in a few works of literature on the method to present its efficiency by calculating wake 
size, wake length, volume, current speed and drag coefficient. For example, Wang et al., [15], Kim et al., [16,17], Woo et al., [14] and Liu and Su [18], to name a few. A relationship normally established on the efficiency index and the ability of the marine species to settle and optimize the energy around the AR.

To examine the wake region, Kim et al., [17] conducted CFD simulations for 24 different artificial reefs (AR) by comparing $A R^{\prime} s$ wake length, deformation and stresses. By using computational fluid dynamics (CFD) Ansys CFX, 3-dimensional (3D) approach was used to observe the distribution of the wake region. It was found that as the AR height increase, the wake length is increased.

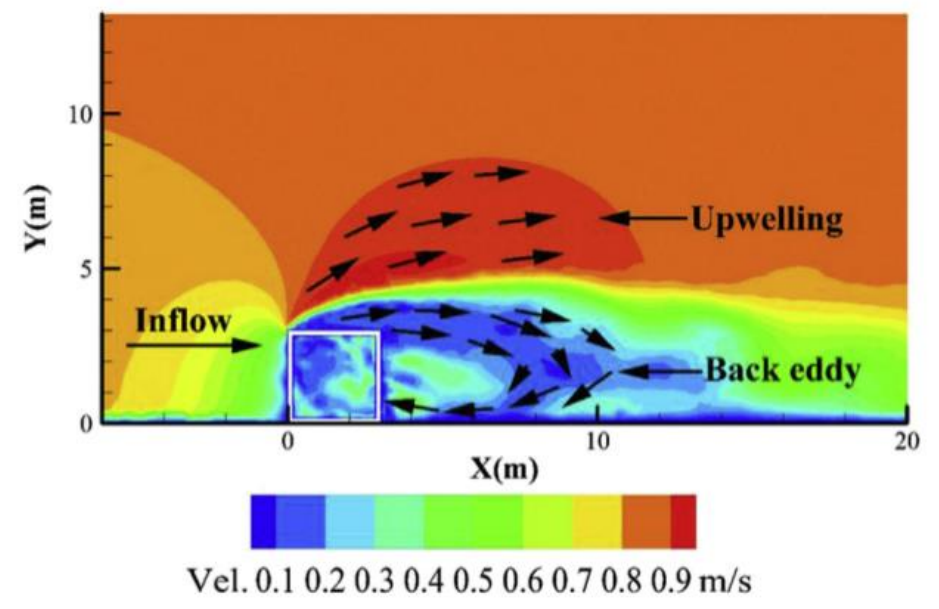

Fig. 1. Velocity magnitude profiles in the wake region [16]

The circulation of the water column around the AR is crucial to ensure its effectiveness. Different approaches mentioned before to measure the performance of the AR. It can be presented using indices of the upwelling and back eddy as an indicator to measure the wake region the AR [15-17]. The number of openings and the size of the opening significantly affects the efficiency index of the artificial reef. This was due to higher permeability occurred when the number and the size of the opening are increase [15].

Potential of artificial reef particularly from the perspective of harbouring biological community within/ around the reefed structure, can be assessed from two key domains, i.e. (1) Present of fish community and (2) Settlement of new benthic communities including corals. The fundamental requirement for the presence of fish communities in the reefed structure is governed by wake characteristic (upwelling, back eddy \& wake) which has been reported by Kim et al., [17], Huang et al., [19], Tang et al., [20] and Wang et al., [15], to name a few. Meaning, all criteria on upwelling, back eddy and wake shall be fulfilled to meet the condition for present of the fish community.

While pressure and the frequency on the artificial reef are critical for the settlement of a new benthic community including corals [13,21-27]. In order to harbor new benthic community, reefed structure has to be satisfied both conditions in the adhesive strength (pressure) and frequency. Failing to meet either one of the conditions will result in low biological potential in the AR. Therefore, coral larvae settlement depends on various important factors such as flow characteristics, vorticity, surface pressure and vibration. These parameters can be considered as physical factors which dealing with the flow around the AR and forces exerted on the structure. To the authors' knowledge, the information on the jacket platform as the RTR is still lacking, thus this paper takes into consideration for these physical parameters which, can significantly investigate the effects of the current flow around the AR of jacket platform using Computational Fluid Dynamics (CFD). 


\section{Methodology}

\subsection{Geometry Configuration and Grid Discretization}

As manifested in Figure 2(a), the conceptual offshore rig structure is modelled in Solidworks composed of 84 numbers of circular cylinders members orientated to $45^{\circ}$ angular configuration. Since the numerical investigation focuses on the application of AR via CFD analysis, design specification and parameters on the circular design structure is not taken into consideration. Hence, the design parameters of the rig structure are modelled at arbitrary to resemble an offshore rig structure. Since the finite volume method (FVM) is applied, cavity region of the body of study is created via Boolean subtract mathematical operation, as shown in Figure 2(b).

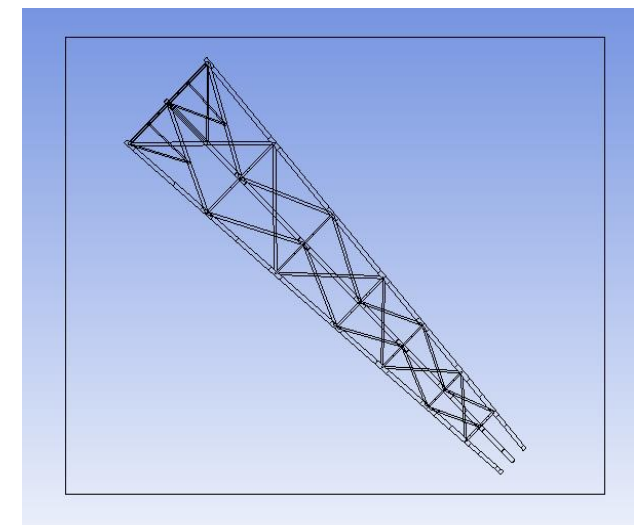

(a)

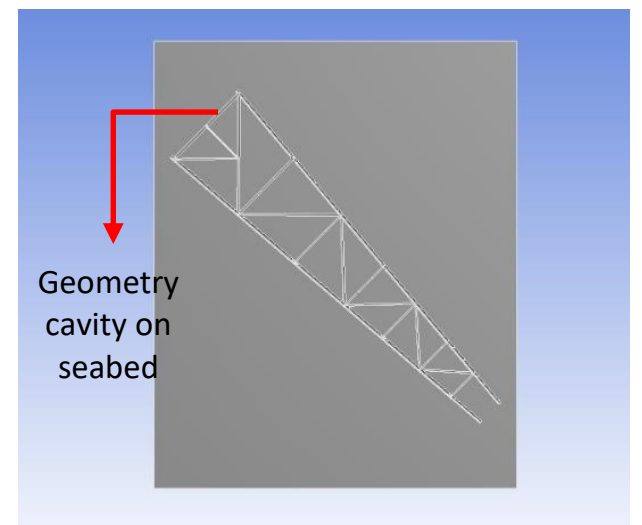

(b)

Fig. 2. (a) Platform configuration (b) Boolean subtract configuration

Since the computational study is conducted under a stationary configuration. The virtual domain is discretized under static mesh configuration via tetrahedrons element type using conformal patch method as shown in Figure 3(a) and Figure 3(b). Due to the complexity of the presented geometry, the virtual topology is discretized using an unstructured grid. In order to save computational load and time of analysis, the platform is discretised without the influence of boundary layer mesh.

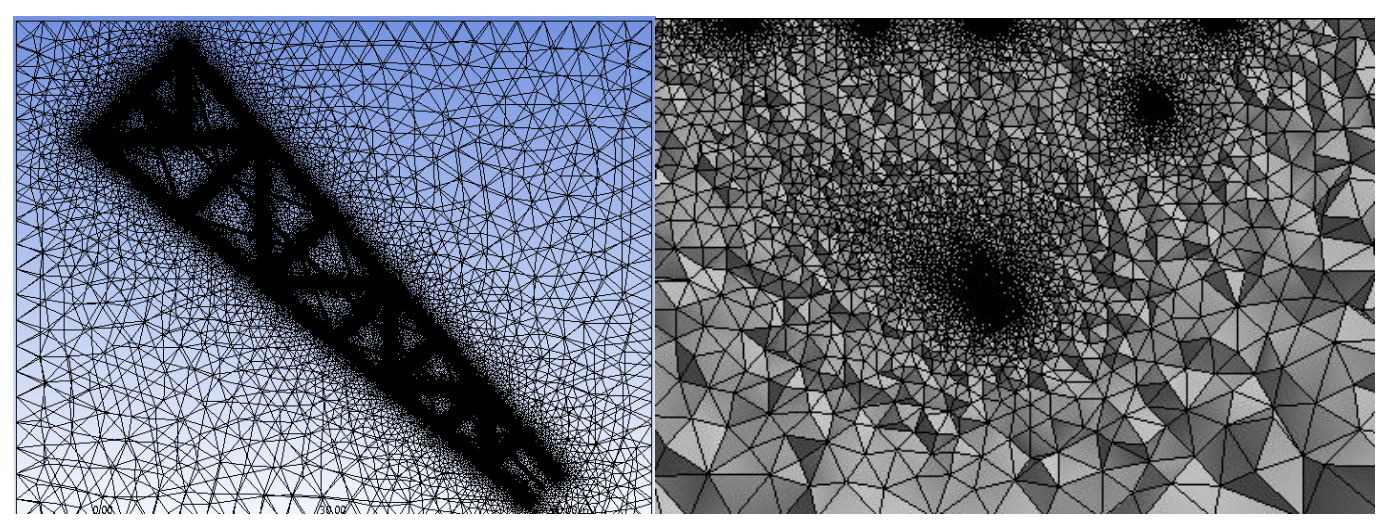

(a) (b)

Fig. 3. Discretized grid configuration: (a) Topside view; (b) isometric view

However, to compensate for the absence of the boundary layer along the wall structure, a denser mesh is mapped along the platform region with growth transition by the ratio of 1.1. The element size is set to $3.2 \mathrm{~m}$ with linear mesh transition and relevance centre to medium. The presented domain is composed of 11665007 number of elements under the linear element order. Table 1 shows the discretized mesh parameters and Table 2 shows the mesh quality parameters. 
Table 1

Discretization grid parameters

\begin{tabular}{ll}
\hline Parameter & Values \\
\hline Mesh scheme & Cell-centred scheme (Fluent) \\
Mesh size & $3.2 \mathrm{~m}$ \\
Number of elements & 11665007 \\
Growth transition & 1.1 \\
Element type & Tetrahedron \\
Discretization Method & Patch conforming method \\
Grid type discretization & Conformal mesh \\
Element order & Linear element \\
\hline
\end{tabular}

Table 2

Mesh metric quality

\begin{tabular}{lllll}
\hline Parameter & Min & Max & Average & Standard deviation, $\sigma$ \\
\hline Orthogonal Quality & 0.11719 & 0.99639 & 0.76926 & 0.11848 \\
Skewness & $1.0438 \times 10-4$ & 0.88281 & 0.22953 & 0.1201 \\
Jacobian ratio & 1 & 1 & 1 & 0 \\
Aspect ratio & 1.1612 & 18.028 & 1.842 & 0.46581 \\
\hline
\end{tabular}

\subsection{Computational Configuration}

In this computational numerical study, the flow field properties of the presented structure were analyzed via FVM approach. Finite volume method or commonly called as box method is widely recommended by researcher due to its robust computational methodology and economical computational load in comparison to other numerical methodologies such as a discrete vortex method (DVM) [28]. In other words, FVM is a procedure of investigating a body under the influence of external flow by means of spatial domain discretization, where the governing partial differential equations are converted to algebraic equations. In order to compute the fluxes of the governing equations across the discretized grid domain, an appropriate numerical model is required to enumerate the flow variables without jeopardizing the reliability of the flow field and without incurring large computational load.

\subsubsection{Boundary conditions}

The platform is simulated at transient mode via pressure-based solver, assuming the density of the flow remain constant throughout the simulation framework using AMD Ryzen $2990 \mathrm{wx} 3.4 \mathrm{GHz}$ (32 processors); 64GB ram. A shown in Figure 4, the domain is initiated with the liquid medium of density $1025.8 \mathrm{~kg} / \mathrm{m}^{3}$ to resemble seawater at the inlet from left to right of the domain with free stream velocity of $1.1 \mathrm{~m} / \mathrm{s}$. Table 3 shows the fluid properties while Table 4 shows boundary conditions setting used in the simulations. The outlet is set as a pressure outlet at 0-gauge pressure. The bounding top and bottom of the domain are set to symmetry at free slip condition type in order to reduce computational power and solid blockage effect $[29,30]$.

Continuity equation

$\frac{d}{d t} \int_{c v} \rho \nu+\int_{c s} v \bullet n \rho d A=0$ 


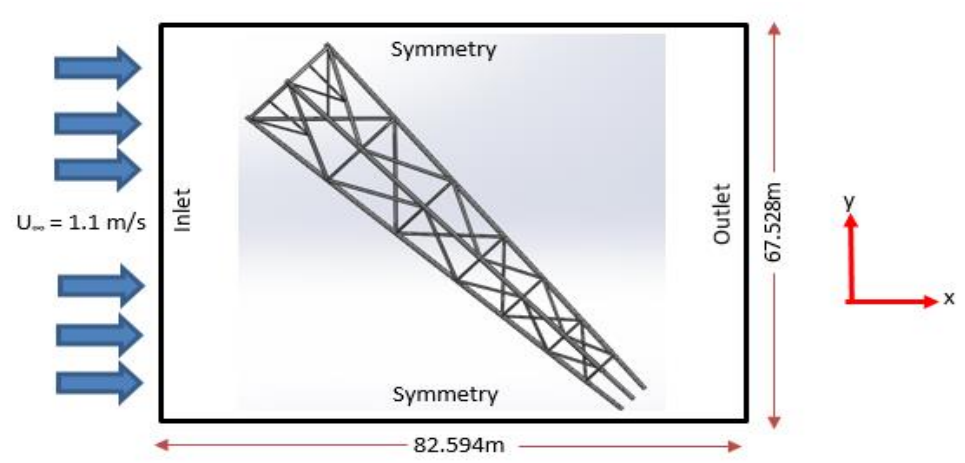

Fig. 4. Boundary conditions setting for the simulation domain

Table 3

Fluid properties

\begin{tabular}{lll}
\hline Parameter & Symbol & Values \\
\hline Density & $\rho$ & $1025.8 \mathrm{~kg} / \mathrm{m} 3$ \\
Dynamic viscosity & $\mu$ & $0.001375 \mathrm{~kg} / \mathrm{m}-\mathrm{s}$ \\
\hline
\end{tabular}

Table 4

\begin{tabular}{lll} 
Boundary condition & & \\
\hline Boundary conditions & Parameters & Values \\
\hline Inlet & U⿺ & $1.1 \mathrm{~m} / \mathrm{s}$ \\
Outlet pressure & Gauge pressure & 0 -pascal \\
Walls & Symmetry & - \\
Platform walls & No-slip condition & - \\
Turbulent intensity & $\mathrm{I}$ & $5 \%$ \\
Turbulent viscosity & $\mu \mathrm{t} / \mu$ & 10 \\
\hline
\end{tabular}

\subsubsection{Solver configuration}

Unsteady Reynolds-averaged Navier-Stokes (URANS) numerical model is utilized to compute the Reynolds stresses of the flow. URANS model operates on the basis of summing the fluctuating and mean components of the flow variables computed by Navier-Stokes equation (NSE) as shown in Eq. (2) [29]. URANS is further classified into three groups of numerical methodology which is linear eddy viscosity, non-linear eddy viscosity, and Reynolds stress model. The most widely used URANS model for an economical approach relative to the two-equation turbulent transport model is linear eddy viscosity. In terms of turbulent transport models, the most common model and computationally economical is $k-\omega$, which is a two-equation turbulent transport model which is $k-\omega$ and $k-\varepsilon$. Meanwhile, SST $k-\omega$ model is widely used because of its ability to accurately compute the adverse pressure regions and flow separation of flow over a body due to its blending functions of the standard $k-\omega$ and $k-\varepsilon[30,31]$.

Reynolds decomposition (URANS)

$u(x, y, z, t)=\bar{u}(x, y, z)+u^{\prime}(x, y, z, t)$

Turbulent kinetic energy, ${ }^{k}$-equation 


$$
\frac{\partial k}{\partial t}+U_{j} \frac{\partial k}{\partial x_{j}}=P_{k}-\beta^{*} k \omega+\frac{\partial}{\partial x_{j}}\left[\left(v+\sigma_{k} \nu_{T}\right) \frac{\partial k}{\partial x_{j}}\right]
$$

Specific rate of dissipation of kinetic energy, ${ }^{\omega}$ - equation

$$
\frac{\partial \omega}{\partial t}+U_{j} \frac{\partial \omega}{\partial x_{j}}=a S^{2}-\beta \omega^{2}+\frac{\partial}{\partial x_{j}}\left[\left(\nu+\sigma_{\omega} \nu_{T}\right) \frac{\partial \omega}{\partial x_{j}}\right]+2\left(1-F_{1}\right) \sigma_{\omega^{2}} \frac{1}{\omega} \frac{\partial k}{\partial x_{i}} \frac{\partial \omega}{\partial x_{i}}
$$

Hence, SST $k-\omega$ is chosen to compute the flow field properties because of its computational economic behaviour. As aforementioned, the objective of this study is to analyze the hydrodynamic parameters relative to the geometry and flow fields, namely, drag and lift coefficient. Hence, the frontal area of the projected area of the platform relative to the configuration is calculated via "projection area module in Fluent". Since the direction of the freestream flow is in the x-direction, the projected area is computed relative to the $x$-direction by means of frontal area. The frontal area of the presented geometry to given angular coordination is $435.4825 \mathrm{~m}^{2}$. In order to save computational load, the flow parameter such as Courant number is set to 60 and COUPLE pressurevelocity scheme is utilized under second-order upwind scheme. The timestep size is set to 0.06 with 1000 iterations of 20 iterations per timestep. Table 5 shows the parameters of solver configuration.

Table 5

Solver configuration

\begin{tabular}{lll}
\hline Solver configuration & Parameters & Values \\
\hline Turbulent transport model & SST $k-\omega$ & Production limiter \\
Reference value & Reference area for lift and drag & $435.4825 \mathrm{~m}^{2}$ \\
Pressure-velocity coupling scheme & COUPLE & - \\
turbulent discretization & Second-order upwind & - \\
Convergence criteria & Residual monitor & $10^{-5}$ \\
Simulation time & Time & $60 \mathrm{sec}$ \\
Timestep & Size & 0.06 \\
Iteration & Size & 10000 \\
Courant Number & $\mathrm{C}$ & 60 \\
\hline
\end{tabular}

Hydrodynamics force components acting on the structure due to vortex shedding are drag and lift forces defined as

$$
\begin{aligned}
& F_{D}=0.5 \rho A U^{2} C_{D} \\
& F_{L}=0.5 \rho A U^{2} C_{L}
\end{aligned}
$$

where $C_{D}$ and $C_{L}$ are the drag and lift coefficients; $D$ is the diameter of the cylinder; $U$ is the flow velocity and where $A$ is usually the frontal area (the area projected on a plane normal to the direction of flow) of the body. The volume rendering needs to be numerated in terms of efficiency index in order to further understand the flow characteristic of upwelling, back eddy and wake velocity. Hence, Eq. (7), Eq. (8) and Eq. (9) were used to numerated the efficiency index which is represented as $I_{u}$ and $I_{\text {backeddy }}[16]$.

Volume-weighted averaged velocity

$\bar{v}=\frac{\int v d V}{\dot{V}}=\frac{1}{\dot{V}} \sum_{i=1}^{n} v_{i}\left|\dot{V}_{l}\right|$ 
Efficiency index, Upwelling

$I_{\text {upwelling }}=\frac{V_{\text {upwelling }}}{V_{A R}}$

Efficiency index, Back eddy

$I_{\text {backeddy }}=\frac{V_{\text {backeddy }}}{V_{A R}}$

\subsection{Grid Sensitivity Study}

In CFD analysis, the discretized spatial configuration is an important criterion in obtaining a stable and reliable numerical result. Since the FVM method is utilized in this study under the unstructured collocated grid domain with the first-order element or linear element tetrahedron elements. The grid has to be analyzed under a different number of elements in order to obtain the suitable grid configuration of which does not cost high computational cost. Hence the grid sensitivity approach is utilized. As manifested in Figure 5, the drag coefficient values decrease as the number of elements rises, which shows the governing relationship of the number of elements to computational time and parameter result.

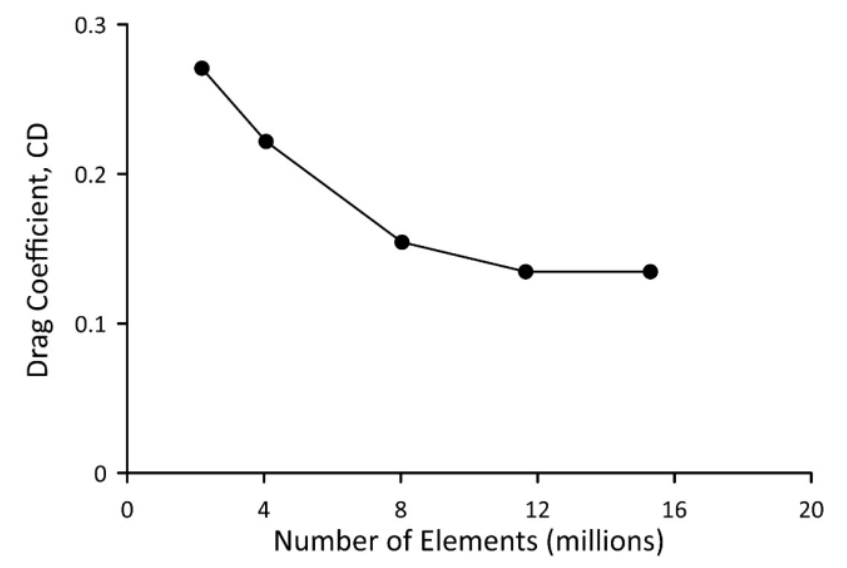

Fig. 5. Drag coefficient on a different number of elements for the mesh sensitivity study

The drag coefficient value reaches a steady state as the number of elements increases. The number of elements in the range of $10-15$ million is taken into consideration due to its stable residual mass imbalance oscillation in comparison to its counterpart. Table 6 shows the value for the parameters extracted from the sensitivity study.

Table 6

Numerical parameters

\begin{tabular}{llll}
\hline Parameter & Symbol & Values & Unit \\
\hline Drag Force & $F_{D}$ & 36417.67 & $\mathrm{~N}$ \\
Drag Coefficient (mean) & $C_{D}$ & 0.134 & \\
Lift Force & $F_{L}$ & 36417.67 & $\mathrm{~N}$ \\
Lift Coefficient (mean) & $C_{L}$ & 0.029 & \\
Shedding Frequency & $f_{v s}$ & 0.06 & $\mathrm{~Hz}$ \\
Strouhal number & $S_{t}$ & 3.8 & \\
\hline
\end{tabular}




\subsection{Directionality}

In order to study the influences of the current directions between the flow and the artificial reef, three (3) dominant direction which are normal, parallel and $45^{\circ}$ from the normal axis were considered. The selection of the dominant flow direction was due to the consideration of streamlining effect and higher frontal area (the area subjected to the flow) for normal and $45^{\circ}$ flow directions, which induced the highest hydrodynamic forces on the structure [5]. Meanwhile, the parallel flow direction was intended to study the wake characteristics for back eddies and upwelling phenomenon. Frontal area, $A$ is an area where one can see the body from the fluid direction.

The notation of the flow directions will be unique for each platform. In a similar note, widely researcher conducts CFD simulation study of external flow relative to $0^{\circ}, 45^{\circ}$ and $90^{\circ}$ based on the projection of the frontal area. Regardless of the study in aerodynamic or hydrodynamic, the flow pattern relative to the incident angle is critical and significant at preliminary at three directions, as shown in Figure 6. However, in specialized case studies specified incident angle is required to study the flow field characteristic such as the angle of attack of airfoil blade, turbomachinery blade in which the angle is studied at an incremental angle. Similar studies on incident angle study on external flow regime can be found in the study by Yoon et al., [32].

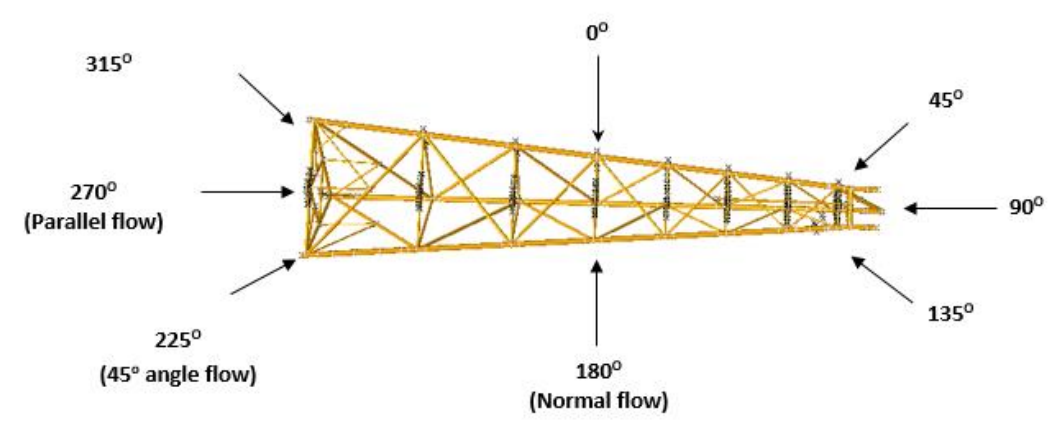

Fig. 6. Direction of current flow for CFD analysis

\section{Results}

\subsection{Pressure Distribution}

Figure 7 shows the static pressure contour regime of the platform. As manifested in the figure, the peak static pressure region of the platform indicated in red contour. It is notable that, the structure the widely enveloped by orange and yellow contour regions, which falls within the regions of the low-pressure region. Table 7 shows the maximum pressure value on the structure for different current flow directions, respectively. The pressure was higher in the normal direction to the flow $\left(0^{\circ} / 180^{\circ}\right)$ as the frontal area is higher compared to other direction. However, since the jacket legs are circular cylindrical structure, the separation of the flow is better compared to other shapes.

Table 7

Comparison of maximum pressure for different directions

\begin{tabular}{ll}
\hline Direction & Max Pressure $(\mathrm{Pa})$ \\
\hline $0^{\circ} / 180^{\circ}$ & $6.58 \times 10^{2}$ \\
$225^{\circ} / 315^{\circ}$ & $6.32 \times 10^{2}$ \\
$45^{\circ} / 135^{\circ}$ & $6.331 \times 10^{2}$ \\
$270^{\circ}$ & $7.15 \times 10^{2}$ \\
$90^{\circ}$ & $6.763 \times 10^{2}$ \\
\hline
\end{tabular}



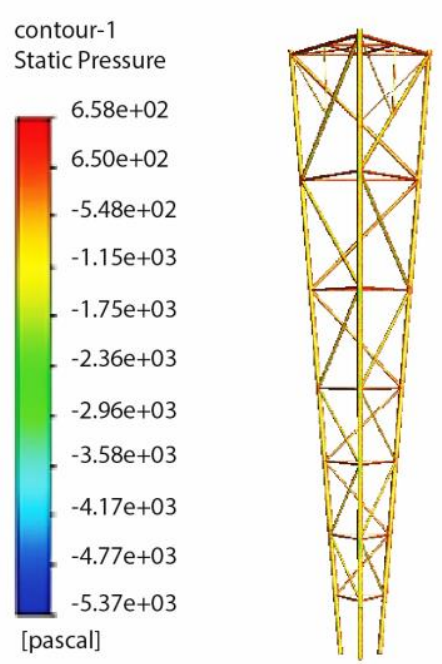

(a)

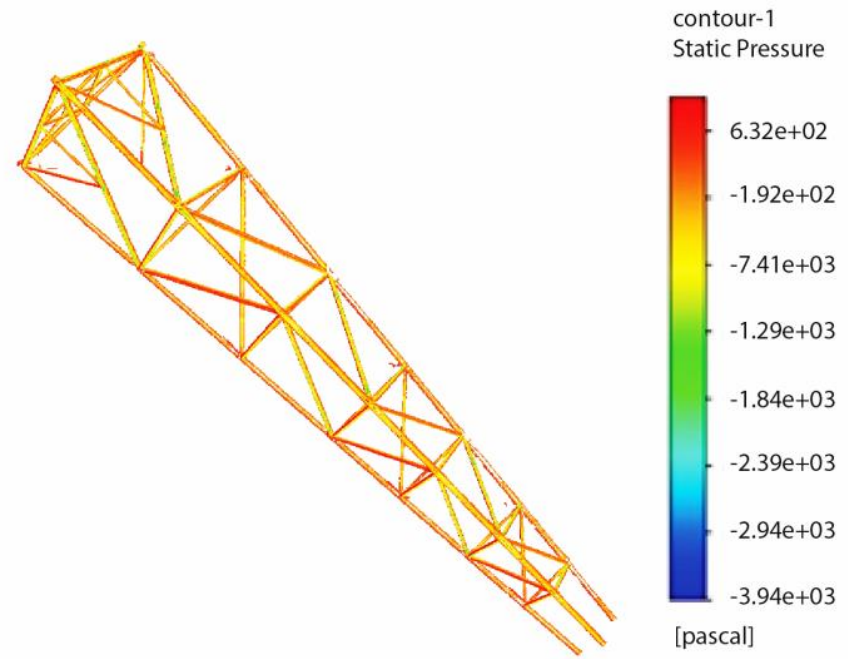

(b)

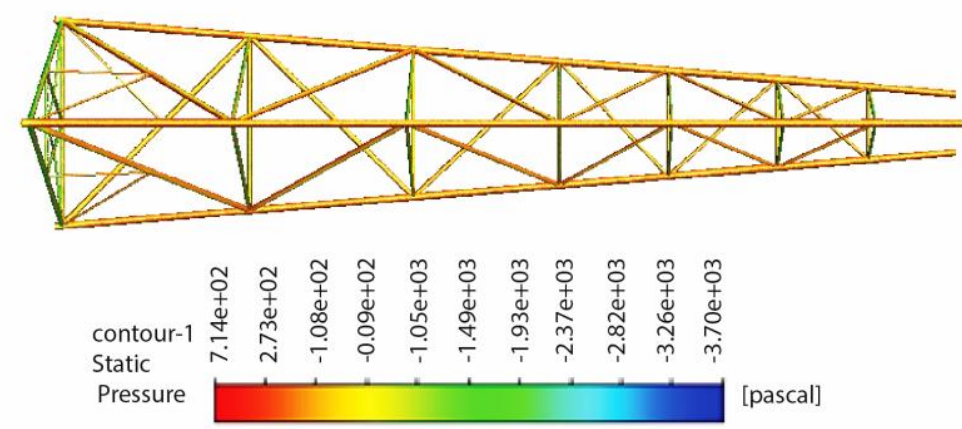

(c)

Fig. 7. Static pressure contours at different current directions. (a) $0^{\circ} / 180^{\circ}$ (b) $225^{\circ} / 315^{\circ}$ (c) $270^{\circ}$

\subsection{Drag and Lift Forces}

The fluctuation of drag and lift coefficients were calculated from the forces acting on the platform boundaries. Table 8 shows the global drag and lift forces/coefficients for the offshore platform subjected to the current flow on the seabed. Results of hydrodynamic coefficients and pressure from the simulations of each directions were used to draw a relationship on the effect of these parameters on the settlement and growth of scleractinian coral. What can be clearly seen in this table is the highest drag force occurred at $0 \% 180^{\circ}$ current direction, normal to the flow. This was obvious since the drag coefficient was expected to be higher as the frontal area is high for the current AR sitting [33]. Meanwhile, lift coefficients show a significant low value which can be neglected.

\section{Table 8}

Hydrodynamics forces on the platform

\begin{tabular}{lllll}
\hline Direction/ Parameter & $\begin{array}{l}\text { Drag Force, FD } \\
(\mathrm{N})\end{array}$ & $\begin{array}{l}\text { Mean Drag Coefficient } \\
\mathrm{C}_{\mathrm{D}(\text { mean })}\end{array}$ & $\begin{array}{l}\text { Lift Force, FD } \\
(\mathrm{N})\end{array}$ & $\begin{array}{l}\text { Mean Lift Coefficient } \\
\mathrm{C}_{\mathrm{L}(\mathrm{mean})}\end{array}$ \\
\hline $0^{\circ} / 180^{\circ}$ & 57810.25039 & 0.314212 & -1199.216757 & -0.00652 \\
$225^{\circ} / 315^{\circ}$ & 36417.67 & 0.134 & 7881.436 & 0.029 \\
$45^{\circ} / 135^{\circ}$ & 36505.77 & 0.134 & -9413.46 & -0.03172 \\
$270^{\circ}$ & 30237.84 & 0.310 & -1253.19 & -0.01284 \\
$90^{\circ}$ & 29850.9 & 0.304 & 118.3866 & 0.01206 \\
\hline
\end{tabular}




\subsection{Vortex Shedding}

Figure 8 illustrates the local vorticity and the wake flow behaviour on the $x$-y plane with an offset of $(-61 \mathrm{~m})$ from the datum plane. As shown in the figure, the flow over the platform body indicated dense flow separation and wide wake region. Due to the orientation of the jacket platform, the flow pattern indicated wake region in which is comparatively different at a various angle of incident. Figuratively speaking, if the platform stays at original configuration and orientation, such flow pattern behaviour can be accepted. However, under the actual circumstance, there is a tendency for the Artificial Reef (AR) to change its orientation due to the ocean current flow and seabed condition, in which comprehensive elaboration can be proved by conducting a scouring analysis. Table 9 shows the frequency and Strouhal number for the AR at different current direction.

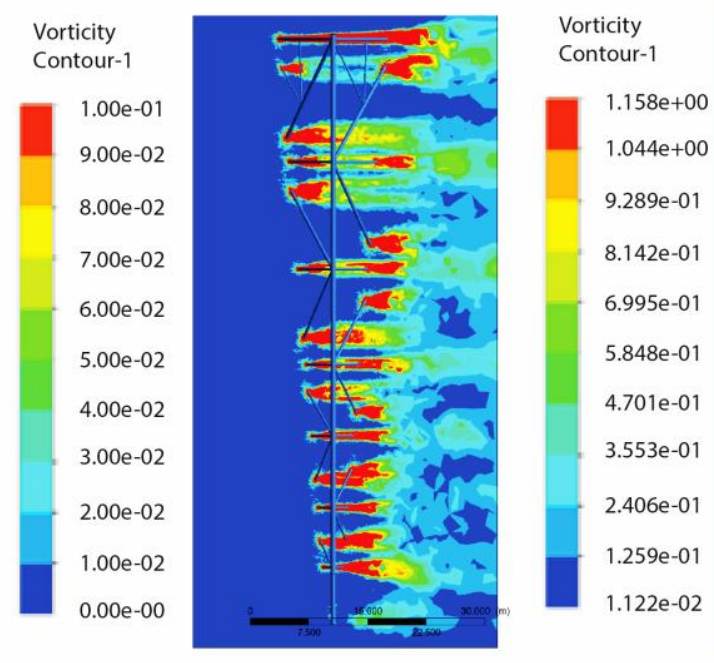

(a)

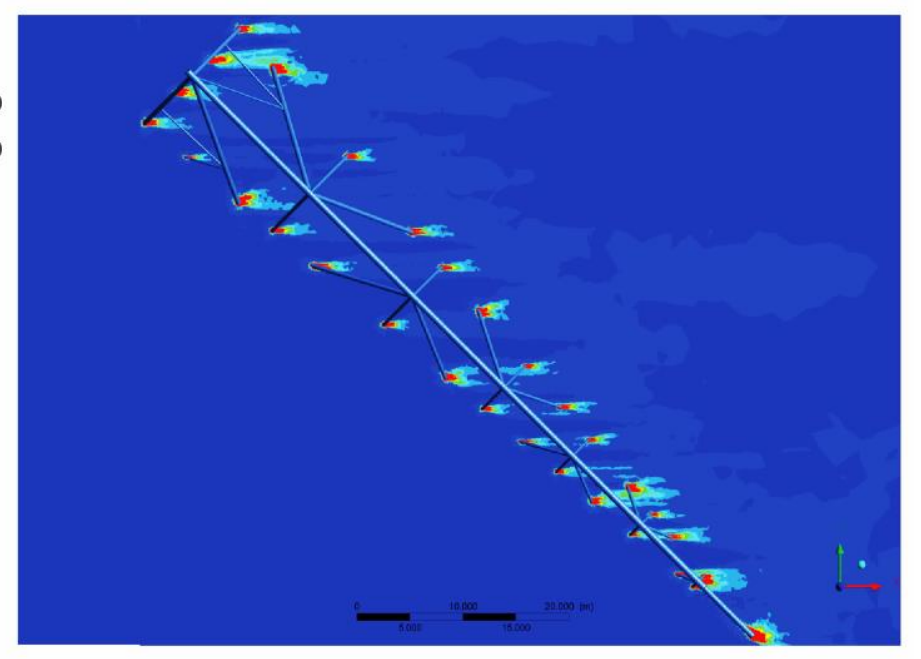

(b)

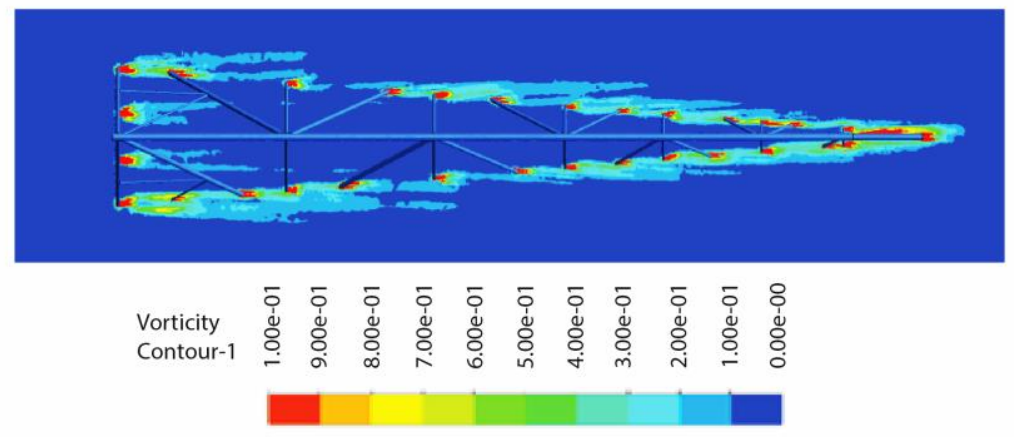

(c)

Fig. 8. Vorticity and wake region on the plane at $-61 \mathrm{~m}$ from the datum. (a) $0^{\circ} / 180^{\circ}$ (b) $225^{\circ} / 315^{\circ}$ (c) $270^{\circ}$

Table 9

Frequency and Strouhal number

\begin{tabular}{lll}
\hline Direction/ Parameter & Shedding Frequency, $f_{v s}$ & Strouhal Number, St \\
\hline $0^{\circ} / 180^{\circ}$ & $0.055 \mathrm{~Hz}$ & 3.784 \\
$225^{\circ} / 315^{\circ}$ & $0.060 \mathrm{~Hz}$ & 3.800 \\
$45^{\circ} / 135^{\circ}$ & $0.060 \mathrm{~Hz}$ & 3.800 \\
$270^{\circ}$ & $0.231 \mathrm{~Hz}$ & 3.762 \\
$90^{\circ}$ & $0.231 \mathrm{~Hz}$ & 3.762 \\
\hline
\end{tabular}




\subsection{Back Eddies and Upwelling}

In order to examine the back eddies and upwelling flow regime with regards to the discretized scheme, Iso-volume domain was separately created in a CFD post under velocity parameter at a range of different values. Based on the volume integral module in Fluent, the volume-weighted average velocity was calculated. Hence, in order to calculate the efficiency index for the artificial reef, the volume of back eddy velocity and upwelling, iso-volume were rendered and calculated in a different range of velocity. Figure 9 shows the views of back eddy velocity volume rendering. Meanwhile, Figure 10 shows the upwelling volume rendering. The efficiency index of the platform was tabulated in Table 10 in order to understand further the flow characteristic of the upwelling and back eddy velocity. The efficiency index was defined as the ratio of the upwelling/back-eddy volumes to the jacket platform volume. It is apparent in the table that the highest upwelling efficiency index is at $0^{\circ} / 180^{\circ}$ current direction. There was an important positive correlation between the drag force and the upwelling volume.

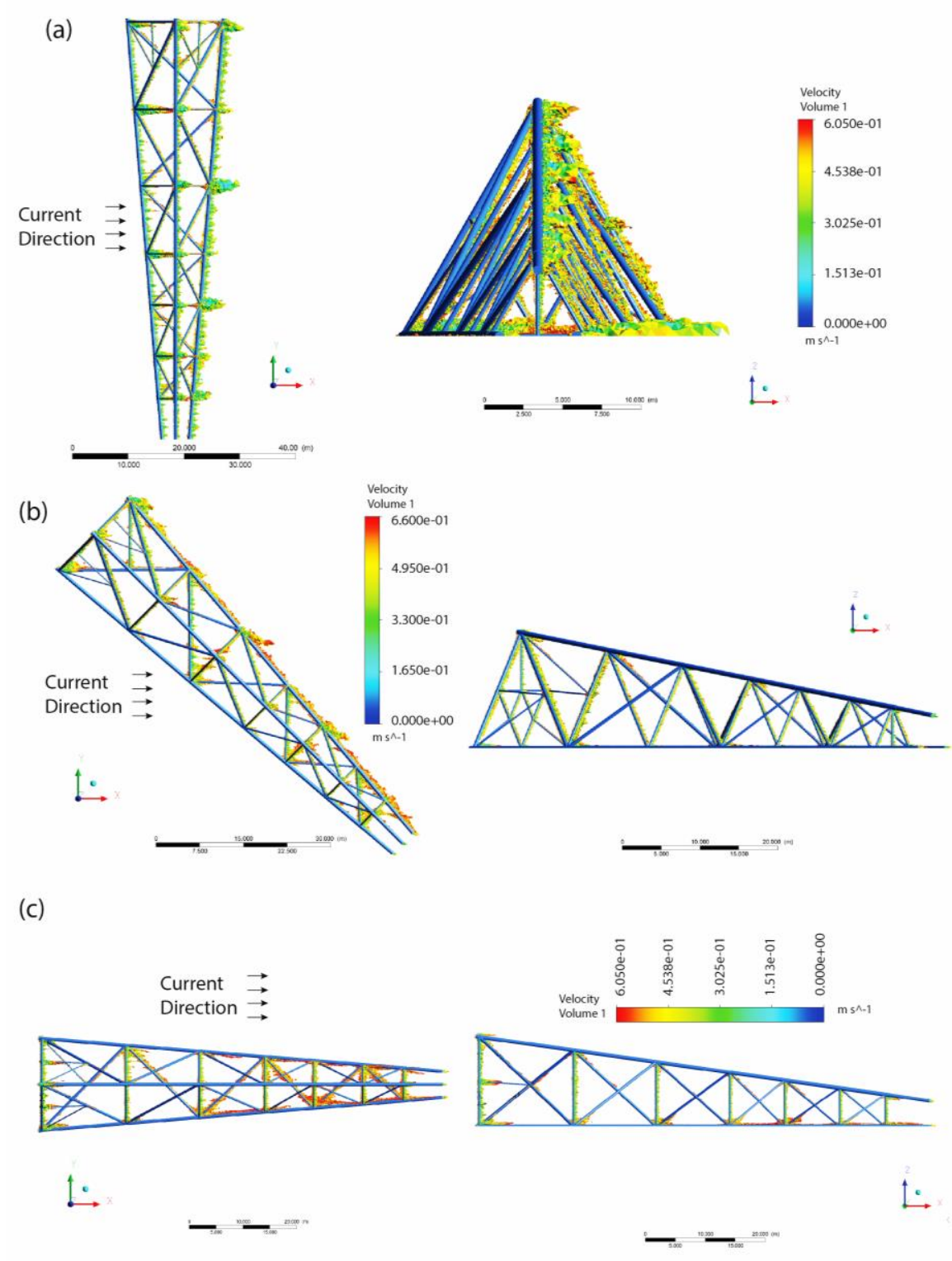

Fig. 9. Back eddies volume profile at different incidence angle. (a) $0^{\circ} / 180^{\circ}$ (b) $225^{\circ} / 315^{\circ}$ (c) $270^{\circ}$. Right: top view, left: side view 
(a)

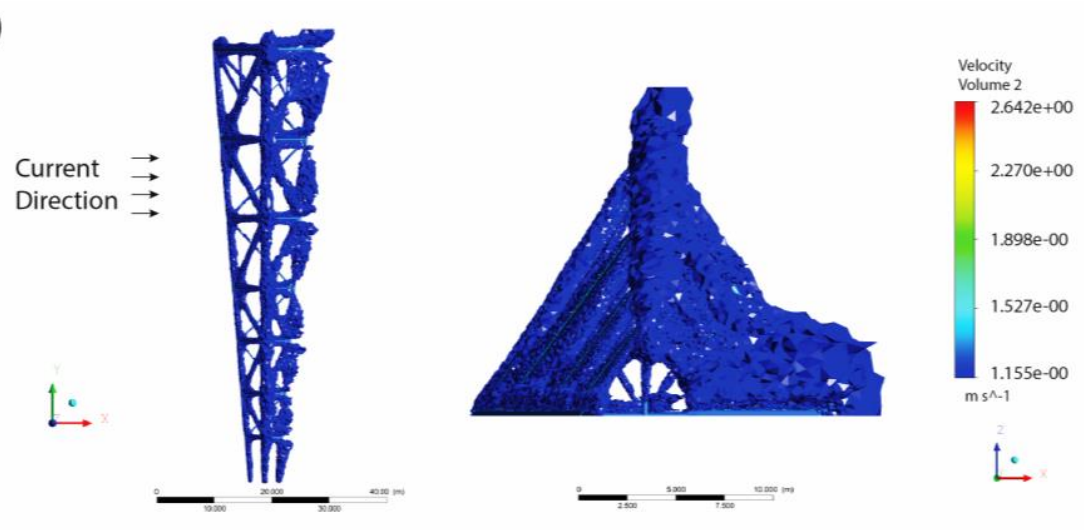

(b)

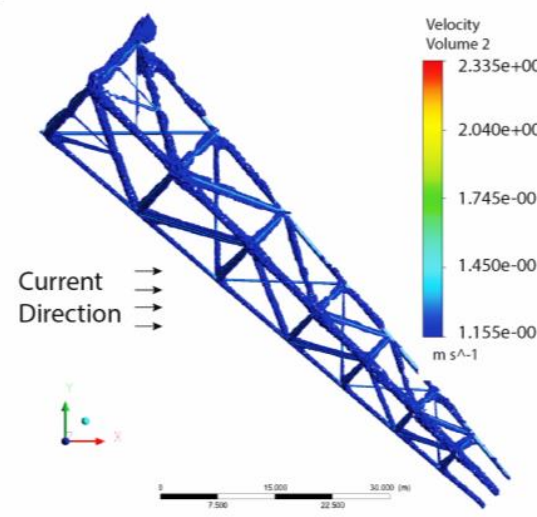

(c)
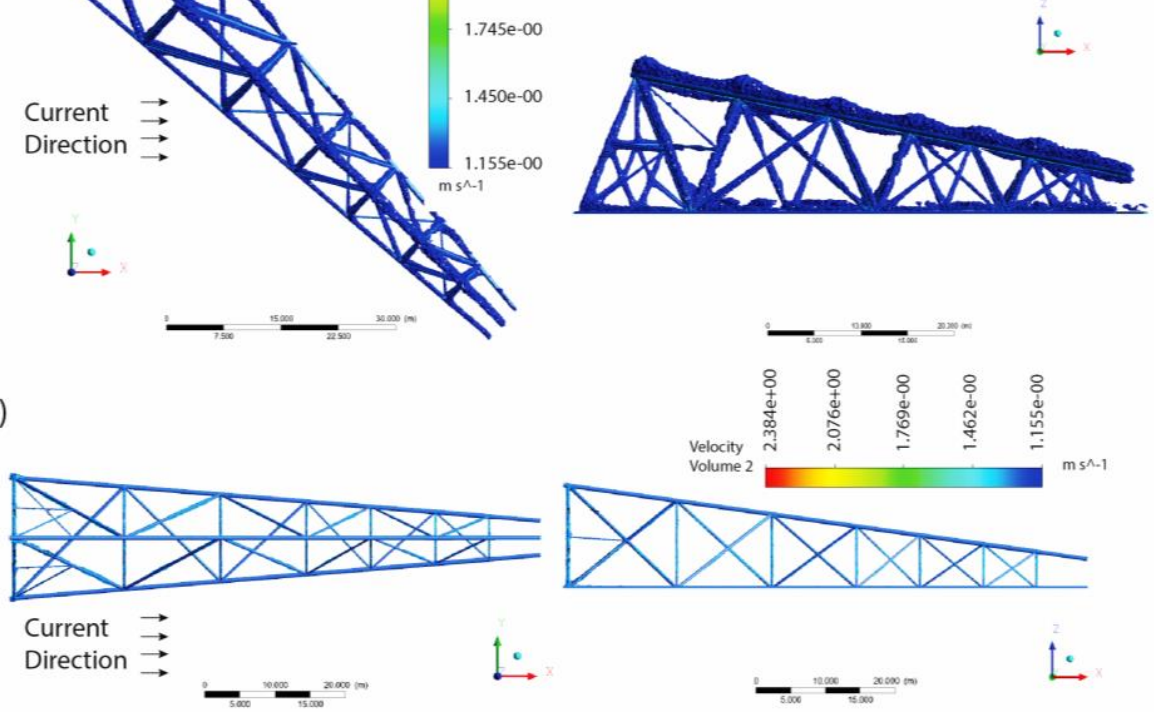

Fig. 10. Upwelling volume profile at different incidence angle. (a) $0^{\circ} / 180^{\circ}$ (b) $225^{\circ} / 315^{\circ}$ (c) $270^{\circ}$. Right: top view, left :side view

Table 10

Efficiency index

\begin{tabular}{lllllll}
\hline Direction & $\begin{array}{l}\text { Upwelling } \\
\text { velocity range } \\
(\mathrm{m} / \mathrm{s})\end{array}$ & $\begin{array}{l}\text { Back eddy } \\
\text { velocity range } \\
(\mathrm{m} / \mathrm{s})\end{array}$ & $\begin{array}{l}\text { Upwelling } \\
\text { volume } \\
\left(\mathrm{m}^{3}\right)\end{array}$ & $\begin{array}{l}\text { Back eddy } \\
\text { volume }\left(\mathrm{m}^{3}\right)\end{array}$ & $\begin{array}{l}\text { Upwelling } \\
\text { efficiency index, } \\
\text { lupwelling }\end{array}$ & $\begin{array}{l}\text { Back eddy } \\
\text { efficiency index, } \\
\text { lbackeddy }^{\circ}\end{array}$ \\
\hline $0^{\circ} / 180^{\circ}$ & $1.155-2.642$ & $0.00-0.605$ & 1288.81 & 17.160 & 9.756 & 0.130 \\
$225^{\circ} / 315^{\circ}$ & $1.155-2.335$ & $0.00-0.605$ & 421.344 & 6.6874 & 3.189 & 0.051 \\
$45^{\circ} / 135^{\circ}$ & $1.155-2.398$ & $0.00-0.605$ & 386.29 & 6.386 & 2.924 & 0.0483 \\
$270^{\circ}$ & $1.155-2.384$ & $0.00-0.605$ & 24.5475 & 7.3460 & 0.186 & 0.056 \\
$90^{\circ}$ & $1.155-2.411$ & $0.00-0.605$ & 22.1204 & 7.062 & 0.167 & 0.05346 \\
\hline
\end{tabular}

This was probably due to a higher frontal area which subsequently generates more vortices and wakes behind the platform. As for the back-eddy efficiency index, a similar direction $\left(0^{\circ} / 180^{\circ}\right)$ is observed for the highest value of 0.130 . Generated values of platform volume and average velocity were tabulated in Table 11. 
Table 11

Platform and simulation generated value

\begin{tabular}{ll}
\hline Parameters & Value \\
\hline Jacket Platform volume $\left(\mathrm{m}^{3}\right)$ & 132.105 \\
Volume weighted average velocity $(\mathrm{m} / \mathrm{s})$ & 1.101508 \\
\hline
\end{tabular}

\section{Conclusions}

The numerical simulation of an artificial reef was presented. Based on the CFD simulation, the result can be concluded as follows

i. Maximum pressure generated on the platform is $7.15 \times 10^{2} \mathrm{~Pa}$ at $270^{\circ}$ current direction, which can be considered low compared to the range of pressure from the literature which is around $10 \times 10^{4} \mathrm{~Pa}$ for biofouling detachment. Dominant pressure on the platform falls in the low range of pressure which is $-1.75 \times 10^{3}$ to $-5.48 \times 10^{2} \mathrm{~Pa}$, which indicates the potential of coral to settle and grow on this particular platform in the current sea condition.

ii. The highest frequency generated from vortex shedding is $0.231 \mathrm{~Hz}$ at $270^{\circ}$ current direction. It shows a lower range of frequency. Low vortex shedding frequency indicates the good sign of the weak chaotic effect, which would be ideal for coral larvae attachment and growth.

iii. The highest upwelling efficiency index is at $0^{\circ} / 180^{\circ}$ current direction, which shows the potential of this platform as an artificial reef at the respective sitting position on the seabed. The upwelling flow field increases fish's biological productivity by increasing the nutrient content. In the meantime, a shaded geometric region spread around and inside the reef can help algae and plankton thrive. So, the reefs can provide shelter from predators and also an abundant source of food.

iv. The highest back-eddy efficiency index was generated at $0^{\circ} / 180^{\circ}$ current direction. The backeddy region usually provides shelter, spawning grounds, rest areas, and a temporary stopover for marine species. However, due to the simple geometry of the jacket platform in this study, less area was provided for marine species to stay and take shelter around the platform.

Finally, both fish community and settlement of new benthic organisms on the artificial reefs are independent of each other. Fish community can be developed surrounding the structure, even without the presence of corals. Likewise, the establishment of new benthic community including corals is independent from the fish community; although coral reefs are habitats of reef fishes. These results suggest that as for present conditions studied, the coral larva can attach and grow on the jacket platform. The jacket platform is proposed to be at normal to the flow ( $180^{\circ}$ direction) as this would generate higher upwelling and back eddies around the jacket platform. This would eventually increase the jacket platform performance as an artificial reef.

\section{Acknowledgement}

This research was funded by a grant from Ministry of Higher Education of Malaysia (FRGS Grant VOT59503).

\section{References}

[1] Bull, Ann Scarborough, and Milton S. Love. "Worldwide oil and gas platform decommissioning: a review of practices and reefing options." Ocean \& coastal management 168 (2019): 274-306.

https://doi.org/10.1016/i.ocecoaman.2018.10.024 
[2] Tsukamoto, K., T. Kawamura, T. Takeuchi, T. D. Beard Jr, and M. J. Kaiser. "Coastal artificial habitats for fishery and environmental management and scientific advancement." In Fisheries for Global Welfare and Environment, 5th World Fisheries Congress, pp. 335-349. 2008.

https://doi.org/10.1111/i.1095-8649.2009.02441 1.x

[3] Sommer, Brigitte, Ashley M. Fowler, Peter I. Macreadie, David A. Palandro, Azivy C. Aziz, and David J. Booth. "Decommissioning of offshore oil and gas structures-Environmental opportunities and challenges." Science of the total environment 658 (2019): 973-981.

https://doi.org/10.1016/i.scitotenv.2018.12.193

[4] Nugraha, R. B. A., R. Basuki, J. S. Oh, II H. Cho, N. Naibaho, Y. Secasari, and L. O. N. Mbay. "Rigs-To-Reef (R2R): A new initiative on re-utilization of abandoned offshore oil and gas platforms in Indonesia for marine and fisheries sectors." In IOP Conference Series: Earth and Environmental Science, vol. 241, no. 1, p. 012014. IOP Publishing, 2019. https://doi.org/10.1088/1755-1315/241/1/012014

[5] Yaakob, Omar B., Yasser M. Ahmed, M. Rajali Jalal, A. A. Faizul, Koh K. Koh, and Tarmizi J. Zaid. "Hydrodynamic Design of New Type of Artificial Reefs." In Applied Mechanics and Materials, vol. 819, pp. 406-419. Trans Tech Publications Ltd, 2016. https://doi.org/10.4028/www.scientific.net/AMM.819.406

[6] Obura, David Obare, Ward Appeltans, Natchanon Amornthammarong, Greta Aeby, Nicholas J. Bax, Joe Bishop, Russel E. Brainard et al. "Coral reef monitoring, reef assessment technologies, and ecosystem-based management." Frontiers in Marine Science 6 (2019): 580.

https://doi.org/10.3389/fmars.2019.00580

[7] Roberts, Louise, and Michael Elliott. "Good or bad vibrations? Impacts of anthropogenic vibration on the marine epibenthos." Science of the total environment 595 (2017): 255-268.

https://doi.org/10.1016/i.scitotenv.2017.03.117

[8] Popper, Arthur N., and Anthony Hawkins, eds. The effects of noise on aquatic life II. New York, NY: Springer, 2016. https://doi.org/10.1007/978-1-4939-2981-8

[9] Tay, Y. C., P. A. Todd, Per Sand Rosshaug, and L. M. Chou. "Simulating the transport of broadcast coral larvae among the Southern Islands of Singapore." Aquatic Biology 15, no. 3 (2012): 283-297. https://doi.org/10.3354/ab00433

[10] Denny, Mark W., and Mark F. Shibata. "Consequences of surf-zone turbulence for settlement and external fertilization." The American Naturalist 134, no. 6 (1989): 859-889.

https://doi.org/10.1086/285018

[11] Sumer, B. Mutlu, and Rolf Deigaard. "Particle motions near the bottom in turbulent flow in an open channel. Part 2." Journal of Fluid Mechanics 109 (1981): 311-337. https://doi.org/10.1017/S0022112081001092

[12] Abelson, Avigdor, and Mark Denny. "Settlement of marine organisms in flow." Annual Review of Ecology and Systematics 28, no. 1 (1997): 317-339.

https://doi.org/10.1146/annurev.ecolsys.28.1.317

[13] Oliveira, Dinis, and Lena Granhag. "Matching forces applied in underwater hull cleaning with adhesion strength of marine organisms." Journal of Marine Science and Engineering 4, no. 4 (2016): 66. https://doi.org/10.3390/jmse4040066

[14] Woo, Jinho, Dongha Kim, Han-Sam Yoon, and Won-Bae Na. "Characterizing Korean general artificial reefs by drag coefficients." Ocean Engineering 82 (2014): 105-114. https://doi.org/10.1016/j.oceaneng.2014.02.025

[15] Wang, Gang, Rong Wan, XinXin Wang, FenFang Zhao, XiaoZheng Lan, Hui Cheng, WeiYao Tang, and QingLong Guan. "Study on the influence of cut-opening ratio, cut-opening shape, and cut-opening number on the flow field of a cubic artificial reef." Ocean Engineering 162 (2018): 341-352. https://doi.org/10.1016/i.oceaneng.2018.05.007

[16] Kim, Dongha, Jinho Woo, Han-Sam Yoon, and Won-Bae Na. " Efficiency, tranquillity and stability indices to evaluate performance in the artificial reef wake region." Ocean Engineering 122 (2016): 253-261.

https://doi.org/10.1016/i.oceaneng.2016.06.030

[17] Kim, Dongha, Jinho Woo, Han Sam Yoon, and Won Bae Na, "Wake Lengths and Structural Responses of Korean General Artificial Reefs." Ocean Engineering 92, (2014): 83-91. https://doi.org/10.1016/i.oceaneng.2014.09.040

[18] Liu, Tsung-Lung, and Dong-Taur Su. "Numerical analysis of the influence of reef arrangements on artificial reef flow fields." Ocean Engineering 74 (2013): 81-89.

https://doi.org/10.1016/j.oceaneng.2013.09.006 
[19] Luiyi, Huang, Cheng Hui, Tang Yanli, Yang Qian, and Wang Xinxin. "Comparison of three ways to assess the influence range of different artificial reefs." International Journal of Engineering and Technology 9, no. 2 (2017): 105. https://doi.org/10.7763/IJET.2017.V9.953

[20] Tang, Yanli, Xiangyu Long, Xinxin Wang, Fenfang Zhao, and Liuyi Huang. "Effect of Reefs Spacing on Flow Field Around Artificial Reef Based on the Hydrogen Bubble Experiment." In International Conference on Offshore Mechanics and Arctic Engineering, vol. 57748, p. V07BT06A017. American Society of Mechanical Engineers, 2017. https://doi.org/10.1115/OMAE2017-61623

[21] Branscomb, E. Sanford, and Dan Rittschof. "An investigation of low frequency sound waves as a means of inhibiting barnacle settlement." Journal of experimental marine biology and ecology 79, no. 2 (1984): 149-154. https://doi.org/10.1016/0022-0981(84)90215-6

[22] Vosburgh, F. "Acropora reticulata: structure, mechanics and ecology of a reef coral." Proceedings of the Royal Society of London. Series B. Biological Sciences 214, no. 1197 (1982): 481-499. https://doi.org/10.1098/rspb.1982.0023

[23] Chamberlain Jr, John A. "Mechanical properties of coral skeleton: compressive strength and its adaptive significance." Paleobiology (1978): 419-435. https://doi.org/10.1017/S0094837300006163

[24] Rodgers, Ku'ulei, Evelyn Cox, and Craig Newtson. "Effects of mechanical fracturing and experimental trampling on Hawaiian corals." Environmental management 31, no. 3 (2003): 0377-0384. https://doi.org/10.1007/s00267-002-2831-3

[25] Baldock, Tom E., Hassan Karampour, Rachael Sleep, Anisha Vyltla, Faris Albermani, Aliasghar Golshani, David P. Callaghan, George Roff, and Peter J. Mumby. "Resilience of branching and massive corals to wave loading under sea level rise-A coupled computational fluid dynamics-structural analysis." Marine pollution bulletin 86, no. 1-2 (2014): 91-101. https://doi.org/10.1016/i.marpolbul.2014.07.038

[26] Madin, Joshua S., Michael J. O'Donnell, and Sean R. Connolly. "Climate-mediated mechanical changes to postdisturbance coral assemblages." Biology Letters 4, no. 5 (2008): 490-493. https://doi.org/10.1098/rsbl.2008.0249

[27] Tunnicliffe, Verena. "The effects of wave-induced flow on a reef coral." Journal of Experimental Marine Biology and Ecology 64, no. 1 (1982): 1-10. https://doi.org/10.1016/0022-0981(82)90064-8

[28] Mohamed Ali, Jaffar Syed, and M. Mubin Saleh. "Experimental and Numerical Study on the Aerodynamics and Stability Characteristics of a Canard Aircraft ." Journal of Advanced Research in Fluid Mechanics and Thermal Sciences 53, no. 2 (2019): 165-174.

[29] Rahman, Mohd Asamudin A., Jeremy Leggoe, Krish Thiagarajan, Mohd Hairil Mohd, and Jeom Kee Paik. "Numerical simulations of vortex-induced vibrations on vertical cylindrical structure with different aspect ratios." Ships and Offshore Structures 11, no. 4 (2016): 405-423. https://doi.org/10.1080/17445302.2015.1013783

[30] Ashwindran, S. N., A. A. Azizuddin, and A. N. Oumer. "A moment coefficient computational study of parametric drag-driven wind turbine at moderate tip speed ratios." Australian Journal of Mechanical Engineering (2020): 1-15. https://doi.org/10.1080/14484846.2020.1714364

[31] Ramponi, R., and Bert Blocken. "CFD simulation of cross-ventilation flow for different isolated building configurations: validation with wind tunnel measurements and analysis of physical and numerical diffusion effects." Journal of Wind Engineering and Industrial Aerodynamics 104 (2012): 408-418. https://doi.org/10.1016/j.jweia.2012.02.005

[32] Yoon, Dong-Hyeog, Kyung-Soo Yang, and Choon-Bum Choi. "Flow past a square cylinder with an angle of incidence." Physics of fluids 22, no. 4 (2010): 043603. https://doi.org/10.1063/1.3388857

[33] Chua Bing Liang, Akmal Nizam Mohammed, Azwan Sapit, Mohd Azahari Razali, Mohd Faisal Hushim, Amir Khalid and Nurul Farhana Mohd Yusof. "Numerical Simulation of Aerofoil with Flow Injection at the Upper Surface." CFD Letters 12, no. 1 (2020): 98-110. 